\title{
Conformable, Stretchable Sensor To Record Bladder Wall Stretch
}

\author{
Stuart Hannah, ${ }^{\dagger}, \S$ Pauline Brige, ${ }^{\dagger}$ Aravind Ravichandran, ${ }^{\dagger}$ and Marc Ramuz ${ }^{*}{ }^{\dagger}$ (1) \\ ${ }^{\dagger}$ Department FEL, Mines Saint-Etienne, Centre CMP, F-13541 Gardanne, France \\ ${ }^{\ddagger}$ Country Centre Européen de Recherche en Imagerie Médicale (CERIMED), Faculté de Médecine, Aix-Marseille Université, 13385 \\ Marseille Cedex 5, France
}

\section{Supporting Information}

ABSTRACT: A soft, conformable, biocompatible strain sensor based on ultra-thin stretchable electronics is reported. The sensor comprises gold thin films patterned on a $50 \mu \mathrm{m}$ thick polyurethane substrate to produce resistive-based strain sensors for monitoring bladder stretch. The sensor responds linearly as a function of strain from 0 to $50 \%$, with an increasing sensitivity as a function of sensor length. The sensor displays good stability with very little hysteresis when it is subjected to cycling between 0 and a maximum strain of $50 \%$, with the largest deviation between 0 and $50 \%$ strain of $\sim 19 \%$ after 100 cycles attributed to the sensor with the longest length $(6 \mathrm{~mm})$ because it physically stretches by a greater distance than sensors with a shorter length. "Breaking" tests on the
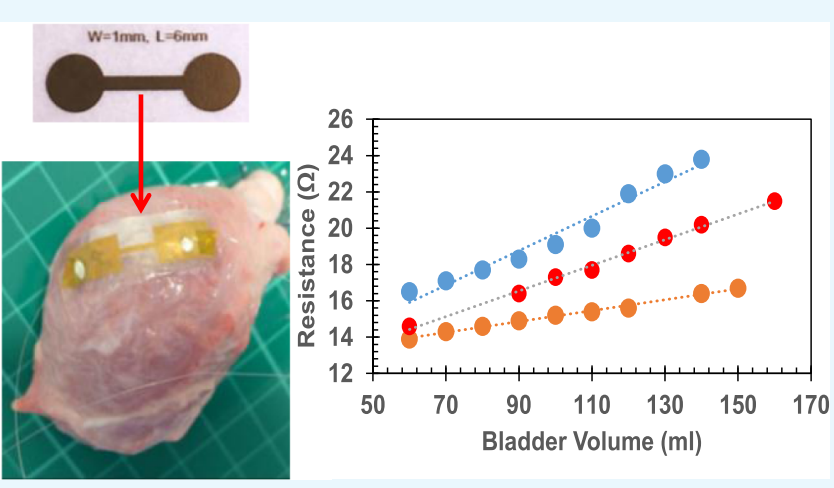
sensor reveal that shorter sensors can withstand higher maximum strains than longer sensors. A biocompatible hydrogel adhesive is used to attach sensors in vitro to the outside wall of a pig's bladder, and sensor performance is studied with respect to repeated bladder filling and emptying to investigate stretch changes. By monitoring bladder stretch and thus volume noninvasively, the sensor provides a route for developing new treatment options for various urological conditions.

\section{INTRODUCTION}

Worldwide, hundreds of millions of people are affected by impaired bladder sensation, with more than 400 million people suffering from an overactive bladder $(\mathrm{OAB})$ alone. ${ }^{1}$ Within Europe, $\mathrm{OAB}$ syndrome affects $\sim 17 \%$ people, with that proportion increasing to $30-40 \%$ for those over the age of 75. ${ }^{2} \mathrm{OAB}$, or indeed, urinary incontinence symptoms, include feeling a sudden and intense need to pass urine (urge incontinence), having to urinate frequently, and even leaking urine before reaching the toilet. ${ }^{3}$ As per survey data of $\mathrm{OAB}$ patients, ${ }^{4}$ these symptoms can have an adverse effect on their daily life: from family, work, and social life to their mental and physical health.

Normally, when the bladder is approximately half-full, we feel the need to pass urine as stretch-sensitive nerve endings in the bladder wall signal to the brain a sensation of fullness. ${ }^{5}$ Most people can hold off from passing urine until convenient, when the bladder muscle (detrusor) contracts. However, with $\mathrm{OAB}$ and after spinal cord injury, the detrusor can contract spontaneously, meaning that patients often inadvertently wet themselves. ${ }^{6}$

There exists a real need to be able to measure bladder stretch. For example, for some patients with $\mathrm{OAB}$, the stimulation of the dorsal genital nerve upon the onset of an urge can suppress undesired detrusor contractions and maintain continence. ${ }^{7}$ However, this technique is only semi effective as stimulation timing can be complicated, and a reliable sensor for monitoring bladder status would be invaluable to alert the patient to the need to stimulate. Spinal cord injury can result in overstretching of the bladder, and a bladder stretch/fullness sensor can substantially improve the quality of life. Furthermore, incontinence is a widespread problem in the aging population, ${ }^{8}$ and a device that can alert them (or their carers) to the need to urinate could be precious.

Often, measurements made in in vivo systems and isolated organs rely on changes in bladder pressure as an indirect index of stretch. ${ }^{9}$ The compliant nature of the bladder means the relationship between pressure and volume is not linear, and therefore, using pressure as an indirect measure of stretch is inadequate. The bladder stretches markedly before the internal pressure changes, ${ }^{10}$ making this method unsuitable for precise bladder stretch/volume measurements. Various sensors currently exist to monitor bladder pressure invasively, exploiting a range of sensing techniques from piezoelectricity, ${ }^{11}$ piezoresistance, ${ }^{12}$ bridging resistors, ${ }^{13}$ and capacitance. ${ }^{14}$ However, the drawback of all of these sensors are that they are invasive, which significantly complicates surgery. They also typically feature complex electronics for either data transmission or power management. ${ }^{15,16}$ We have demonstrated a simple, novel, non-invasive method to measure bladder stretch directly without the need for complex electronics, which has

Received: October 1, 2018

Accepted: December 7, 2018

Published: January 24, 2019 
a

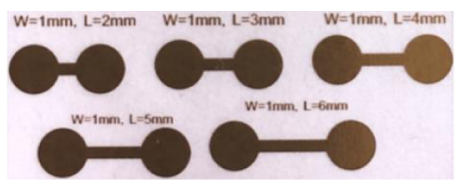

b (i)

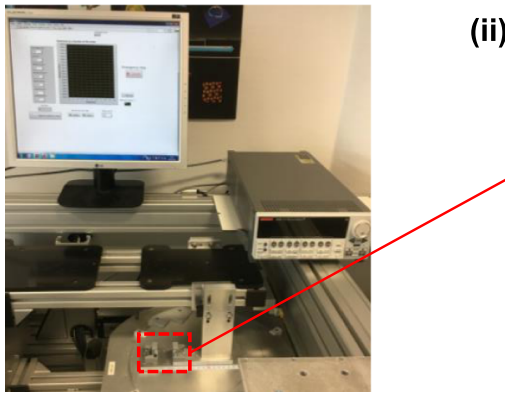

(ii)

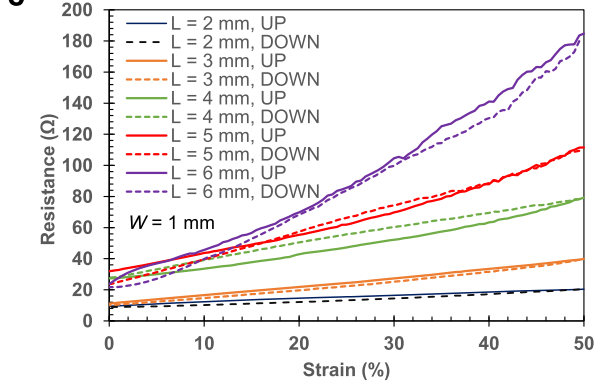

d

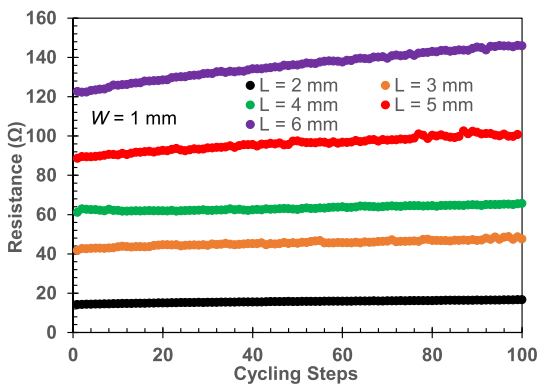

e

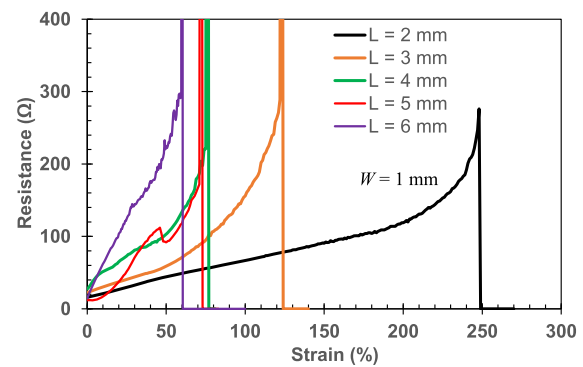

Figure 1. (a) Laser-patterned stretchable strain sensors on a $50 \mu \mathrm{m}$ thick polyurethane film. Sensor dimensions: $W=1 \mathrm{~mm}, L=2,3,4,5$, and 6 mm. (b) Apparatus used to characterize sensor electrical and mechanical measurements: (i) $x-y$ table and (ii) sensor under initial and stretched conditions. (c) $\mathrm{Cr} / \mathrm{Au}$ sensor resistance as a function of strain for sensors featuring dimensions $W=1 \mathrm{~mm}$ and $L=2,3,4,5$, and $6 \mathrm{~mm}$. (d) $100 \times$ cycling tests performed on $\mathrm{Cr} / \mathrm{Au}$ sensors. Graph shows plot of resistance at $50 \%$ strain only. (e) Sensor-breaking tests on devices featuring $W=1$ $\mathrm{mm}$ for $L=2,3,4,5$, and $6 \mathrm{~mm}$.

the potential to significantly enhance the current state-of-theart as well as the wider field of clinical medicine.

Stretchable and flexible electronics ${ }^{17}$ has fantastic but, to date, underexploited potential within biomedical science. By definition, stretchable electronic devices can be stretched out and restored back to their original state without significant deterioration in device performance. ${ }^{18-22}$ Because of the flexible/stretchable nature of such electronics and, increasingly, their biocompatibility, devices involving the human body have been realized, including wearable technology to monitor patient vital signs, biosensors, sensor arrays for in situ perspiration analysis, flexible and stretchable conductors, multifunctional devices for heart arrhythmia therapy, and flexible and foldable electrodes to map brain activity.

Many research groups have focused on the development of flexible sensors for various robotic or medical purposes, ${ }^{21,30}$ realizing these on flexible substrates, such as polyethylene- terephthalate and polyimide (PI). However, these devices would benefit from being stretchable for applications involving the body. Stretchable tactile sensors have been realized previously using pressure conductive rubber, ${ }^{31}$ whereby the resistance of the rubber changes in response to the applied pressure. Ultrasensitive, stretchable strain sensors based on fragmented carbon nanotube papers have also been realized with a gauge factor of over $10^{7}$ at $50 \%$ strain. ${ }^{32}$ For humanmotion detection, stretchable strain sensors based on conductive hydrogels have been created. $^{33}$ A biologically inspired pressure sensor network was developed on silicon islands connected by stretchable wires ${ }^{34}$ and holds promise from e-skin to smart vehicles.

Numerous flexible/stretchable sensors for implantable biomedical applications exist. Flexible electronics for biosignal monitoring, featuring organic thin-film transistors and a biocompatible gel interface have been realized. ${ }^{35}$ By implanting 
the device directly onto the surface of a rat's heart, an electrocardiogram is amplified by the OTFT circuit in vivo. Moreover, an organic, ultra-conformable, biocompatible neural interface array has been developed, which can record action potentials from superficial cortical neurons without penetrating the surface of the brain. ${ }^{36}$ Dissolvable films of silk fibroin have been used to create ultrathin $(2.5 \mu \mathrm{m})$, conformal, biointegrated electronics on a meshed PI substrate to perform in vivo, neural mapping experiments on feline animal models. ${ }^{37}$ A similar system was developed using a class of silicon electronics to map cardiac electrophysiology under bending and stretching. ${ }^{38}$ Stretchable electronics have been used previously to attempt to monitor bladder stretch/volume noninvasively, but with limited success. In one scenario, a polypyrrole-based strain sensor was fabricated to measure bladder volume ${ }^{39}$ and subsequently tested on a balloon model. However, the sensor fabrication was complex and responded only linearly within the range $20-40 \%$ strain.

We present a sensor with key advantages, including its noninvasive nature, its simplicity, and its biocompatibility, rendering it suitable for in vivo applications. The sensors have been fully characterized as stand-alone devices under stretch. In addition, we present the steps taken to ensure effective, biocompatible adhesion between the sensor and the bladder. Finally, sensor performance as a function of bladder stretch is assessed using an isolated pig's bladder in vitro. The results provide a simple and effective method to investigate changes in bladder stretch and pave the way to record the long-term behavior of local microscale changes in bladder tone (micromotions), which can provide a route to develop new treatment options for patients with $\mathrm{OAB}$ or urge incontinence. This work pioneers some of the first research into bladder health monitoring by using noninvasive, stretchable electronic sensor systems.

\section{RESULTS AND DISCUSSION}

2.1. $\mathrm{Cr} / \mathrm{Au}$ Sensors. Figure $\mathrm{la}$ shows the $\mathrm{Cr} / \mathrm{Au}$ sensors fabricated on polyurethane film according to the procedure outlined subsequently. Figure $1 \mathrm{~b}$ shows the process of sensor characterization using the $x-y$ motion table under the initial and stretched conditions. Resistance as a function of strain for various sensor lengths is presented in Figure 1c for sensors featuring $W=1 \mathrm{~mm}$ and $L=2,3,4,5$, and $6 \mathrm{~mm}$. Figure $1 \mathrm{~d}$ presents results of cycling tests performed on sensors with the same dimensions as Figure 1c for 100 cycles between 0 and $50 \%$ strain. Finally, Figure 1e shows results of "breaking" tests performed on the sensors, to assess their maximum stretch capability and investigate the effect of $L$. Some additional supporting graphs can be found in the Supporting Information (Figure S1).

From Figure 1c, by stretching from 0 to $50 \%$ strain, sensor resistance increases linearly, and sensitivity $(S)$ defined as the change in resistance per degree of strain increases with increasing $L$. Referring to the UP ramps, for $L=2 \mathrm{~mm}, S=$ $0.21 \Omega / \%$ strain, whereas for $L=6 \mathrm{~mm}, S=3.18 \Omega / \%$ strain, approximately a 15 -fold increase. The increase in $\mathrm{Cr} / \mathrm{Au}$ sensor resistance sensitivity with increasing $L$ is expected because the initial resistance at $0 \%$ strain is higher with increasing $L$. Sensitivity for each $L$ can be found in the Supporting Information. Hysteresis is smaller for smaller $L$, but in every case, the sensors show repeatable behavior between 0 and $50 \%$ strain. The appearance of cracks in the gold depend on the absolute value of stretch; that is, for $50 \%$ strain, sensors with a length of $2 \mathrm{~mm}$ will stretch by $1 \mathrm{~mm}$, whereas $6 \mathrm{~mm}$ sensors will stretch by $3 \mathrm{~mm}$, ultimately leading to the production of more cracks and increasing the resistance and hysteresis.

To investigate the repeatability of the devices, the sensors were cycled between 0 and 50\% strain for 100 cycles to replicate a natural situation of repeated bladder filling and emptying. From Figure 1d, as sensor $L$ increases, the overall resistance at $50 \%$ strain increases too. In addition, hysteresis increases for increasing $L$. For $L=2 \mathrm{~mm}, R_{0}=14.1 \Omega$, and $R_{100 \text {-cycles }}=16.6 \Omega$, there is an increase of $\sim 16 \%$ over 100 cycles. Conversely, $L=6 \mathrm{~mm}, R_{0}=122.7 \Omega$, and $R_{100 \text {-cycles }}=$ $145.9 \Omega$ give an increase of $\sim 19 \%$ over the cycling range. From Figure 1e, the sensor breaking point (where resistance begins to increase exponentially) reduces as sensor length increases; therefore, sensors with smaller $L$ can be stretched to greater maximum strain. The sensor breaking point for $L=6 \mathrm{~mm}$ is $\sim 59 \%$ strain, whereas it is $\sim 240 \%$ for $L=2 \mathrm{~mm}$, over four times higher.

When the Au films are stretched, cracks begin to appear in the metal layer. At smaller strains, these cracks appear at the edges of the film only, ${ }^{40}$ meaning that larger $W$ sensors will have more area in the center of the film without cracks and therefore require more stretching before breaking. At higher strain, these edge cracks continue to grow across the film, perpendicular to $L$, and produce a dramatic increase in sensor resistance. ${ }^{40}$ Photographs of crack formation in the $\mathrm{Cr} / \mathrm{Au}$ film on PU as a function of stretch between 0 and $100 \%$ can be found in Figure S1c of the Supporting Information.

Because longer $L$ sensors will physically stretch more in distance for the same percentage strain compared to smaller $L$ sensors, there will be more opportunities for cracks to appear in the film, and hence, the "breaking" point appears lower. Overall, sensors featuring smaller $L$ show better performance in terms of hysteresis and breaking point; however, a trade-off exists, because higher length devices feature far greater resistance sensitivity, making them more attractive for a sensing application. Greater sensitivity is critical for sensor application on the bladder, where the sensor must remain sensitive to small changes in bladder stretch. Therefore, to benefit from this requirement, $6 \mathrm{~mm}$ was chosen as the optimum length of the sensor for this application. After some preliminary tests, a sensor width of $1 \mathrm{~mm}$ was chosen as the fabrication standard to ensure that regardless of sensor length, the sensor can withstand a strain of at least $50 \%$, more than satisfactory compared to the actual strain developed when the sensor is attached to the bladder wall. The sensor dimensions were chosen for the following reasons. First, the overall dimension in the millimeter range was guided by the dimensions of a pig or human bladder, as the ultimate end application. Furthermore, the sensors were attached by hand to the bladder, and for readout, a gold wire was attached manually to the sensor contact pad via silver paint. The sensors were designed to have a suitable size for manipulation in a clinical setting by a medical professional. Laser patterning of the sensors was chosen over other techniques, such as photolithography or printing, because laser patterning is a quick, cost-effective, solvent-free, and low-temperature process. The laser was used to ablate metal from a previously deposited $\mathrm{Cr} /$ $\mathrm{Au}$ layer.

2.2. Biocompatibility Steps and Balloon Model. To attach $\mathrm{Cr} / \mathrm{Au}$ sensors to the bladder, various glues were tested to adhere the sensor to a balloon (Table S6, Supporting Information). The majority provided very good adhesion, but 
a

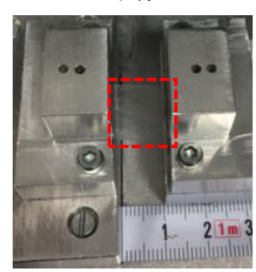

b

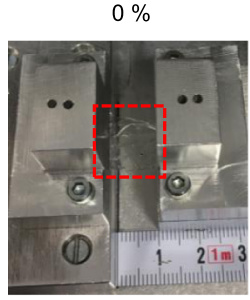

$60 \%$

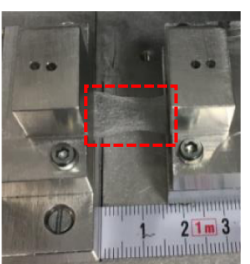

$160 \%$

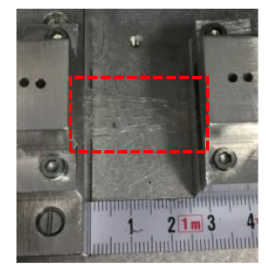

c (i)

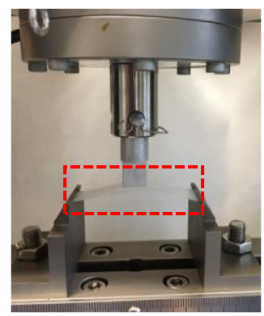

$120 \%$

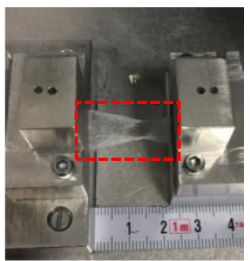

$320 \%$

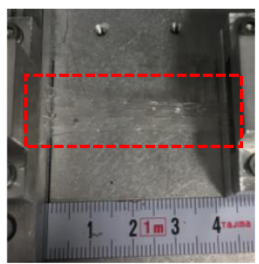

(ii)

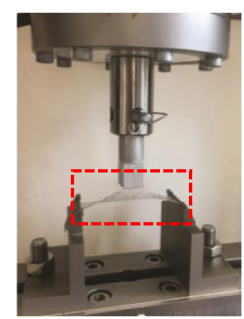

$180 \%$

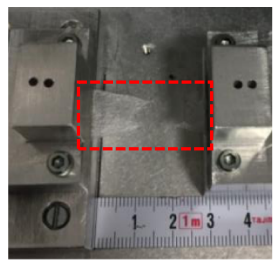

$480 \%$
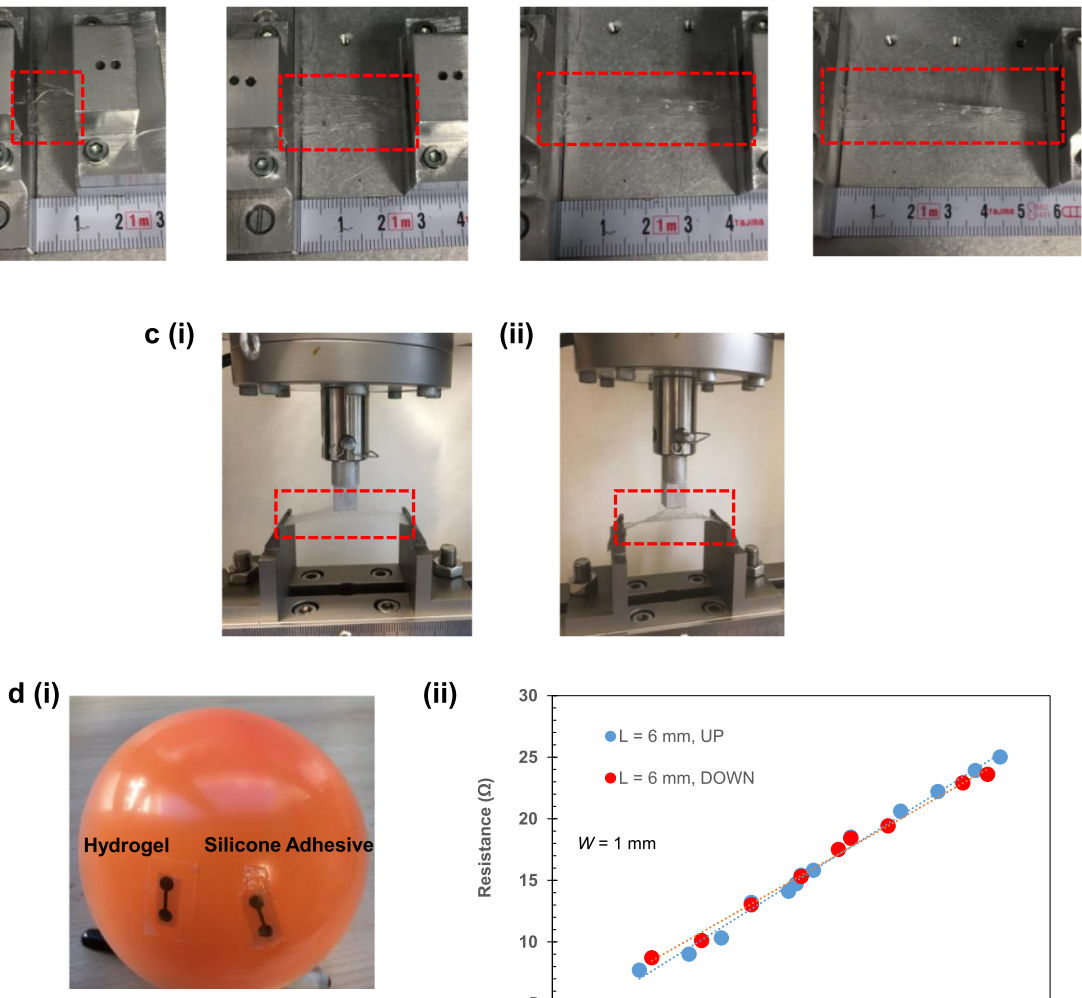

(ii)

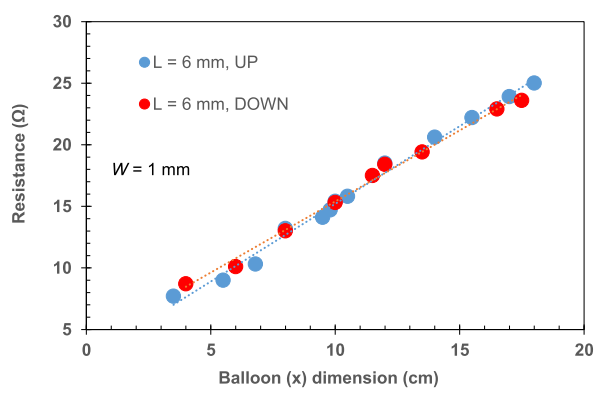

Figure 2. (a) Effect of stretching on biocompatible hydrogel adhesive to $180 \%$ strain. (b) Effect of stretching on biocompatible silicone adhesive to $480 \%$ strain. (c) (i) Hydrogel film under compressive extension test and (ii) silicone adhesive film under compressive extension test. (d) (i) Cr/Au sensors attached to a balloon surface with hydrogel and silicone adhesives. (ii) $\mathrm{Cr} / \mathrm{Au}$ sensor resistance as a function of stretch for a sensor featuring dimensions $W=1 \mathrm{~mm}$ and $L=6 \mathrm{~mm}$ attached to a balloon surface using Polymer Science INC hydrogel adhesive.

did not stretch well when the balloon was inflated/deflated and were also non-biocompatible. Therefore, a transparent silicone sealant (Pattex) was investigated as a first step toward a biocompatible solution. The adhesive adhered the polyurethane well to the balloon and was able to stretch under inflation. However, it takes over 3 hours to fully dry; therefore, with surgical expediency in mind, a biocompatible adhesive was sought, which could dry quickly. A silicone gel/acrylic trilaminate (PS-2066) and a medical-grade hydrogel (PS1446) from Polymer Science INC were chosen for this purpose. According to their respective datasheets, the hydrogel has previously been used in applications ranging from advanced wound care, burn care, transdermal drug delivery, and wearables to device fixation, whereas the silicone adhesive has also been used for scar therapy, surgical draping, and incontinence care and in various healthcare products. To comply with the $\mathrm{Cr} / \mathrm{Au}$ sensor stretching capability up to $\geq 50 \%$ strain, the two adhesives were tested by stretching mechanically using the $x-y$ table. Figure $2 \mathrm{a}$,b show the effect of stretching on the hydrogel and silicone adhesive films, respectively, between the initial position and 180 and $480 \%$ strain, respectively. As each adhesive is stretched from its initial (relaxed) position up to $\sim 60 \%$ strain, the films retain their form and can withstand the mechanical deformation. However, at $\sim 90 \%$, there exists a real difference in the behavior of the two films; it appears that the holes in the silicone adhesive aid its stretchability. This could be related to the holes providing a smaller overall surface area under strain, meaning that the integrity of the entire sample remains better until a much higher strain value. Structurally, the silicone adhesive remains intact and, in fact, can continue to be stretched, until finally breaking at a maximum strain of $\sim 483 \%$. Conversely, the hydrogel starts to break at around $90 \%$ and finally breaks completely at $200 \%$. However, both can stretch significantly more than the $\mathrm{Cr} / \mathrm{Au}$ sensors, making either of them suitable.

In addition to the stretchability of each adhesive, it is important to note their relative adhesion strength to ensure effective attachment to the bladder. According to datasheets, the silicone adhesive has a peel-test adhesion strength of 1.4 $\mathrm{N} / 25 \mathrm{~mm}$, whereas the hydrogel has stronger adhesion, at 5.4 
a (i)

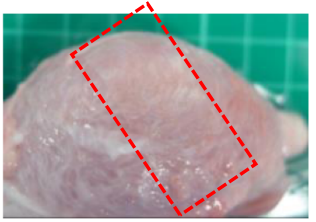

b (i)

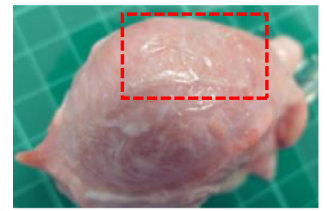

(ii)

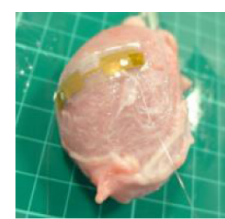

(ii)

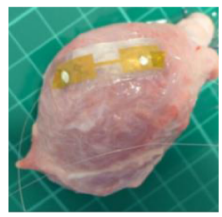

(c)

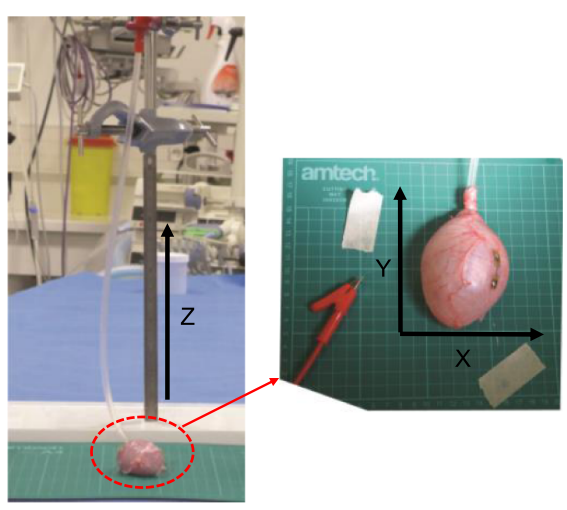

d (i)

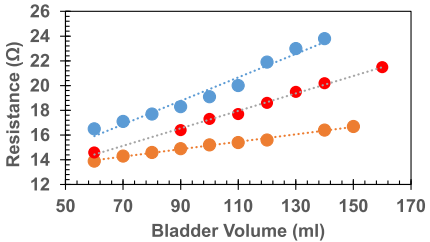

(iv)

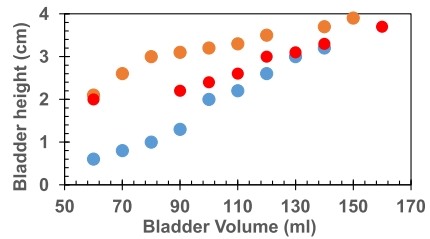

(ii)

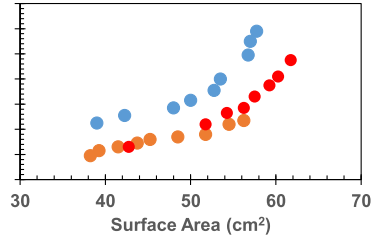

Vertical placement (neck side) taped securely

- Diagonal placement, taped securely

- Vertical placement, tape on base (iii)

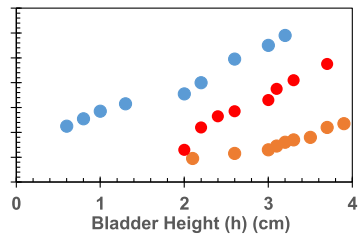

(v)

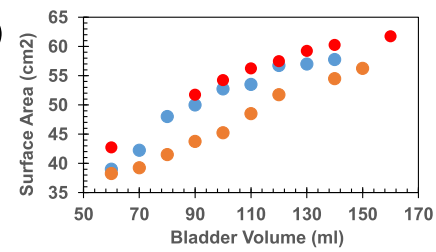

Figure 3. (a) (i) Hydrogel adhesive attached to the bladder surface and (ii) Cr/Au sensor attachment via the hydrogel. (b) (i) Silicone adhesive attached to the bladder surface and (ii) $\mathrm{Cr} / \mathrm{Au}$ sensor attachment via the silicone adhesive. (c) Measurement setup showing $\mathrm{Cr} / \mathrm{Au}$ sensor $(L=6$ $\mathrm{mm}, W=1 \mathrm{~mm}$ ) attached to the surface of a pig's bladder. The photographs show the setup devised to monitor sensor dimensions as a function of bladder volume. (d) (i) Sensor resistance as a function of bladder volume. (d) (ii) Resistance as a function of surface area. (d) (iii) Resistance as a function of height. (d) (iv) Bladder height as a function of volume. (d) (v) Surface area as a function of volume for three Cr/Au sensors attached to the wall of a pig's bladder using Polymer Science INC hydrogel adhesive.

$\mathrm{N} / 25 \mathrm{~mm}$. Using a compressive extension test, mean values of $1.38 \mathrm{~N}$ at $15.6 \mathrm{~mm}$ and $1.35 \mathrm{~N}$ at $13 \mathrm{~mm}$ were measured for the hydrogel and silicone adhesive films, respectively. Figure $2 \mathrm{c}$ shows representative photographs of the tests performed.

Before testing the sensors in vitro on a pig's bladder, a proof of concept was devised using a latex balloon to mimic natural bladder behavior of filling and emptying. Figure $2 \mathrm{~d}(\mathrm{i})$ shows two sensors attached to the surface of a balloon, one attached using the silicone adhesive and the other using the hydrogel. From Figure $2 \mathrm{~d}(\mathrm{ii})$, as the balloon is inflated with air and expands to an $x$-plane dimension $\sim 18 \mathrm{~cm}$, sensor resistance increases from $\sim 8$ to $25 \Omega$, equivalent to a sensitivity of 1.26 $\Omega / \mathrm{cm}$. Upon deflation, the resistance decreases. The sensor displays no hysteresis over this measurement range because the level of stretch of the sensor in this scenario is significantly smaller than the stretch performed on the stand-alone sensor previously. These results are in agreement with the sensor behavior reported in Figure 1c, and provide promising results for successive in vitro testing.

2.3. In Vitro Testing of Sensor. After validation using the balloon, the sensors and biocompatible adhesives were tested in vitro using a pig's bladder. The pig's bladder was obtained from a freshly euthanized pig and was cleaned before use. Figure $3 \mathrm{a}(\mathrm{i})$,(ii) show the hydrogel adhesive attached to the bladder surface and a $\mathrm{Cr} / \mathrm{Au}$ sensor attached to the bladder via the hydrogel, respectively. Similarly, Figure 3b(i),(ii) show the silicone adhesive and its sensor attachment to the bladder surface. Figure $3 \mathrm{c}$ shows the bladder measurement setup devised.

Ultimately, the hydrogel was chosen over the silicone adhesive as the preferred biocompatible adhesive for a number of reasons. First, the hydrogel was far easier to manipulate and could be easily peeled off from its polyethylene/polyester liners, whereas the silicone adhesive was harder to remove from the polypropylene and polycarbonate liners. This is an extremely important consideration because the future application of this device would require manipulation by a surgeon, where quick and effective handling is critical in a clinical setting. Secondly, the sensor attached to the bladder using the hydrogel adhered more strongly and could stretch better as a function of bladder filling and emptying compared to the sensor attached via the silicone adhesive. The hydrogel remained completely flat on the bladder surface, unlike the silicone adhesive, which had a tendency to curl at the edges and wrinkle over time, perhaps because of the effect of moisture. These observations are in contrast to the adhesion strength measured on the two films previously (Figure 2c); however, there will undoubtedly be disparity between the adhesion force measured on a metal clamp and when attached to tissue.

Figure $3 \mathrm{~d}$ presents results of three $\mathrm{Cr} / \mathrm{Au}$ sensors attached to the bladder surface using the hydrogel. Figure $3 \mathrm{~d}(\mathrm{i}),(\mathrm{ii})$,(iii) show sensor resistance as a function of bladder volume, surface area, and height, respectively, and Figure $3 \mathrm{~d}(\mathrm{iv}),(\mathrm{v})$ show 
bladder height and surface area as a function of bladder volume, respectively. Data showing volume as a function of bladder $x$ and $y$ dimensions and dimensions as a function of sensor resistance can be found in the Supporting Information (Figure S3). Results of tests performed on two other pigs' bladders can be seen in Figure S4, Supporting Information

As seen in Figure $3 \mathrm{~d}(\mathrm{i})$, as bladder volume is increased in 10 $\mathrm{mL}$ increments from the initial $60 \mathrm{~mL}$, sensor resistance increases linearly, regardless of sensor placement or adhesion method. The greatest sensitivity of $0.1 \Omega / \mathrm{mL}$ is obtained for the sensor placed vertically between the bladder neck and the base, whereas the sensor placed diagonally shows the lowest sensitivity at $0.03 \Omega / \mathrm{mL}$. As expected, from Figure $3 \mathrm{~d}(\mathrm{iv}),(\mathrm{v})$, as the volume of the bladder increases from 60 to $\sim 160 \mathrm{~mL}$, both bladder height and surface area increase. Therefore, as the surface area (ii) and the height (iii) increase, sensor resistance increases. Resistance increasing as a function of bladder volume is expected because it agrees with results shown previously on the balloon model (Figure $2 \mathrm{~d}$ ). The reason for some discrepancy between bladder height and surface area as a function of volume between the three experiments is bladder handling. During the experimental setup, the bladder position will undoubtedly change, and because the bladder is highly non-conformal, the height and surface area are prone to change from run to run.

For the sensor placed vertically and taped securely, over this $80 \mathrm{~mL}$ increase in bladder volume, the resistance changes by $7.3 \Omega$. We can correlate this change in resistance to the change in resistance seen for the stand-alone sensor with the same dimensions from the strain test in Figure 1c. For the UP ramp only, this change in resistance on the bladder corresponds to a $2.3 \%$ strain, significantly less than the capability of the sensor $(\geq 50 \%)$. Over this range, the $x$-dimension of the bladder increases from 5 to $8.3 \mathrm{~cm}, \Delta x=3.3 \mathrm{~cm}$. Referring to the balloon model, for the same $\Delta x$, the sensor resistance changes by $2.6 \Omega$, which correlates in Figure $1 \mathrm{c}$ to a strain of $0.8 \%$. This strain is similar to that found on the bladder; however, the difference can be attributed to sensor placement. On the balloon, the sensor was placed vertically, closer to the neck of the balloon, whereas on the bladder, although the sensor was again placed vertically, but nominally toward the bottom side of the bladder (away from the neck). In one instance, the sensor was placed vertically on the neck side of the bladder, where it is clear that for the same bladder volume, there is a more considerable change in bladder height, and therefore resistance, compared to with placement closer to the bottom. Comparing the two sensors that were both securely taped, the only difference in the setup was the placement position on the bladder. It appears that the sensor placed vertically on the neck side of the bladder has around 3 times greater sensitivity than the same sensor placed diagonally across the line from the neck to the base, toward the bottom side of the bladder. Figure $3 \mathrm{~d}$ also concludes that the greater the height and surface area, the greater the sensitivity of the sensor. Finally, there appears to be no correlation between sensor taping method and resistance, confirming that placement of the sensor is the only factor that produces a change in resistance sensitivity as a function of volume, meaning that provided the same sensor position is chosen for all experiments, the behavior should be the same.

In this study, the bladder volume was initially prefilled to 60 $\mathrm{mL}$; then up to a further $100 \mathrm{~mL}$ was gradually added in $10 \mathrm{~mL}$ increments. As shown previously, over the tested bladder volume range, the change in sensor resistance corresponds to a strain of $2.3 \%$. For a typical human adult, the bladder is capable of holding up to around $600 \mathrm{~mL}$ of urine, although the urge to urinate is often experienced around $150 \mathrm{~mL} .{ }^{41}$ Therefore, this study has demonstrated a sensor more than capable of withstanding linear and reproducible behavior within a typical bladder volume of interest $(\leq 150 \mathrm{~mL})$, which also has the capacity to withstand maximum volume $(\sim 600 \mathrm{~mL})$, if necessary, demonstrated by linear, repeatable sensor behavior up to a strain of $50 \%$.

Now that the sensor has been demonstrated in practice using an in vitro porcine model, the next step is to move to an in vivo model to verify its effectiveness on a live animal. In vivo testing using a rat will require a number of new considerations, including appropriate surgical techniques, sensor placement, signal readout methods, and interfacing the sensor with the central nervous system. However, the design of the sensor demonstrated and its inherent biocompatibility will ensure that the complexity around addressing these challenges will be reduced significantly. The approach will maintain the simplicity already demonstrated and will provide adequate strain sensitivity to enable effective in vivo measurements to truly provide a basis for monitoring bladder volume efficiently using a stretchable electronics-based approach.

\section{CONCLUSIONS}

We have produced a soft, biocompatible, conformable sensor using stretchable electronics to monitor bladder stretch noninvasively. The simple device consists of $\mathrm{Cr} / \mathrm{Au}$ thin films patterned on stretchable polyurethane, to produce resistive strain sensors. Sensors of varying dimensions were produced, and sensor resistance showed a linear dependence as a function of strain from 0 to $50 \%$, with very little hysteresis. Sensitivity is highest for $L=6 \mathrm{~mm}$, at $3.18 \Omega / \%$ strain, which is around 15 times higher than the sensitivity for $L=2 \mathrm{~mm}$, at $0.21 \Omega / \%$ strain. Smaller $L$ sensors displayed better stability over 100 cycles, and the "breaking" point is higher for shorter $L$ devices.

A biocompatible hydrogel film (Polymer Science INC, PS1446) was found to be the most suitable for application on the bladder because it is easy to handle, water resistant, hypoallergenic, resistant to bacteria, stretchable up to a strain of $200 \%$, and has an adhesion of $1.38 \mathrm{~N}$ at $15.6 \mathrm{~mm}$. Highsensitivity $\mathrm{Cr} / \mathrm{Au}$ sensors with $L=6 \mathrm{~mm}$ were attached to the bladder wall, and resistance was measured as a function of bladder volume as the bladder wall stretched. A maximum sensitivity of $0.1 \Omega / \mathrm{mL}$ was observed for a sensor placed in a vertical position, directly between the bladder neck and the base.

Our approach is advantageous to other systems to monitor bladder pressure found in the literature, such as a mini-invasive approach, ${ }^{42}$ whereby despite its small size, complex electronics are still required to be inserted within the inner lining of the bladder wall, leading to possible patient discomfort over prolonged periods. To avoid implantation of the sensor directly into the bladder, a noninvasive approach has been developed, ${ }^{43}$ which exploits an ultrasound bladder vibrometry (UBV) method to monitor changes in the bladder wall. The approach has provided promising results; however, one drawback of the UBV method concerns the estimation of bladder wall elasticity and thickness, which, if estimated based on poor imaging, could be a limiting factor. As mentioned in the Introduction, one of the closest approaches to our device to monitor bladder volume uses a polypyrrole-based 
stretchable electronics system. ${ }^{39}$ However, this sensor appears to show a linear response within a strain range of $20-40 \%$, whereas stand-alone measurements of our sensor show a linear behavior up to at least $50 \%$ strain.

The key advantage of our sensor over similar devices in the literature, however, lies in its simplicity. The fabrication simply involves the patterning of metal layers on a stretchable PU substrate. Because the sensor relies solely on changes in resistance, it does not require any complex circuitry to read out the data, unlike a system exploiting capacitance ${ }^{14}$ or piezoelectricity ${ }^{11}$ might. It is also fully biocompatible, because it is composed only of $\mathrm{Cr} / \mathrm{Au}$ (with $\mathrm{Cr}$ as an adhesion layer for gold evaporation), polyurethane, and a biocompatible hydrogel adhesive.

This study paves the way toward the development of a sensor system to address challenges including $\mathrm{OAB}$ in a noninvasive manner and presents a crucial step toward implantable urological health-monitoring systems of the future.

\section{EXPERIMENTAL SECTION}

4.1. Cr/Au Sensors-Fabrication. A $5 \mathrm{~nm}$ thick adhesion layer of chromium $(\mathrm{Cr})$ at a rate of $0.1 \AA / \mathrm{s}$, followed by a layer of $\sim 150 \mathrm{~nm}$ thick gold $(\mathrm{Au})$ at $1 \AA / \mathrm{s}$, was deposited using vacuum thermal evaporation (Edwards Auto 500) on a $50 \mu \mathrm{m}$ thick stretchable polyurethane (PU) film (DelStar technologies INC, ref EU50). Chromium featured as an adhesion layer to aid gold attachment to the PU film. The thickness of gold was carefully selected after several preliminary tests, which found that $150 \mathrm{~nm}$ was a reasonable thickness to ensure good stretching performance and relatively low Au crack formation within the range of interest. Prior to metal deposition, the polyurethane film was subjected to a $5 \mathrm{~min} \mathrm{O}_{2}$-activation treatment using reactive ion etching (Oxford Instruments PlasmaLab 80 Plus) to activate its surface. Chromium/gold ( $\mathrm{Cr} / \mathrm{Au}$ ) films were laser patterned (LPKF Laser) to produce sensors featuring lengths $(L)(L=2,3,4,5$, and $6 \mathrm{~mm})$ with a width $(W)$ of $1 \mathrm{~mm}$ (Figure $1 \mathrm{a})$.

4.2. Cr/Au Sensors-Characterization. The sensors were characterized using a dedicated $x-y$ motion table featuring simultaneous electrical and mechanical recording associated with a Keithley 2636A. Electrical measurements, displacement, and data collection were synchronized using a homemade LabView script. Sensors were characterized according to their resistance, measured as a function of tensile strain (nominally $0-50 \%$ ) at a stretching speed of $100 \mu \mathrm{m} / \mathrm{s}$ in a sweep mode (increase and decrease stretch) to investigate hysteresis. Cycling tests between 0 and 50\% strain were performed for 100 cycles to investigate the stability of the sensor under prolonged usage. Sensor resistance was recorded between 0 and $50 \%$ strain, and before stretching, the initial position of the $x-y$ table clamps were adjusted to ensure sample tautness (flat substrate). In every case, at least five sensors of each dimension were tested, which show typical results. Different sensors were used to perform different experiments; for example, the sensors used to produce Figure $1 \mathrm{c}$ are different to those used in (d) and (e), which accounts for the subtle differences measured in resistance initially and at $50 \%$ strain, even for sensors of the same length.

4.3. Biocompatibility Steps. To ensure biocompatibility for subsequent in vitro testing on the bladder, Au was chosen as the main metal layer for the sensors, which has the added advantage of having high conductivity. Sensors were produced on breathable, transparent medical-grade polyurethane/ure-
thane/TPU film to ensure compatibility with living tissue. To interface the sensors with the bladder, a biocompatible adhesive was required to maintain adhesion between the lightweight polyurethane sensor substrate and the dimensionally changing and uneven bladder surface. Two adhesive films were chosen after rigorous testing (Polymer Science INC): a perforated silicone gel/acrylic trilaminate $(d=0.22 \mathrm{~mm})$ consisting of a high-adhesion silicone gel skin contact adhesive $\left(\mathrm{PS}-2066^{44}\right)$ a medical-grade hydrogel $(d=1.2 \mathrm{~mm})$ for use in fixation applications (PS-1446 ${ }^{45}$ ). According to the manufacturer, each film has passed cytotoxicity, primary dermal, and Buehler sensitization biocompatibility tests. The films are also water resistant, hypoallergenic, and resistant to bacteria, which make them particularly suitable for application on the bladder. To ensure effectiveness as an adhesive for a stretchable electronics application, the mechanical robustness of each film was tested by stretching using the $x-y$ table. To verify the adhesion of the two biocompatible films for our application, the adhesion strength of each film was tested by performing a "compressive extension test" (Instron 3365), whereby the force required to remove the adhesive from a metal clamp was measured. The adhesion force was recorded over 5 separate cycles in both cases, and a mean value was taken.

4.4. Balloon Model Setup and Characterization. Two $\mathrm{Cr} / \mathrm{Au}$ sensors $[W=1 \mathrm{~mm}, L=6 \mathrm{~mm}$ (for optimum sensitivity)] were attached to the balloon surface using the silicone adhesive and hydrogel adhesive films, ensuring the whole sensor was taped to the adhesive. Upon placement, the sensors were pre-stretched slightly to ensure good contact between the sensor and the adhesive; therefore, measurements were taken of the relative change in sensor resistance $(\Delta R)$ as a function of balloon stretch, measured as the change in balloon size as it inflates/deflates with air in the $x$-plane. A Keithley $2636 \mathrm{~A}$ ohmmeter was used to measure the resistance of the hydrogel-attached sensor only as a function of balloon inflation between the initial attachment position $(x$-plane $=3.5 \mathrm{~cm})$ and the maximum inflation position of $x=18 \mathrm{~cm}$ (Figure $2 \mathrm{~d}(\mathrm{ii})$ ). Resistance was measured for both UP and DOWN sweeps (inflation and deflation) to investigate hysteresis and was recorded using thin $\mathrm{Al}$ wires attached via conductive silver paint (RS Components) to the sensor contact pads to ensure that the sensor was measured in an identical fashion every time.

4.5. In Vitro Testing Setup and Characterization. Experiments were performed using bladders of male and female pigs, aged between 5 and 6 months. The pig breeds used were either Pietrain or Landrace. The study was approved by the Animal Care Committee of Aix-Marseille University (ethical committee CE71) and was conducted according to European Union Directive 2010/63/EU and the recommendations of the Helsinki Declaration. Experiments were performed in an authorized laboratory (A1305532) by trained and competent researchers. A $60 \mathrm{~mL}$ syringe via a $50 \mathrm{~cm}$ long piece of tubing was attached to the neck of the bladder via stitching to allow bladder filling and emptying with water. Figure $3 c$ shows the devised setup featuring a sensor attached to the bladder surface using the hydrogel adhesive. To measure the changing size of the bladder, it was placed on a measurement mat to monitor dimensional changes in the $x$ and $y$-planes. An overhead camera on a tripod was used to capture $x$ and $y$ movements, whereas changes in the $z$-direction (bladder height) were recorded using a ruler placed in line with another camera. To provide a suitable bladder surface 
area for sensor attachment, the bladder was initially prefilled with $60 \mathrm{~mL}$ water before device placement. Initially, two sensors $(L=6 \mathrm{~mm}, W=1 \mathrm{~mm})$ were attached to the bladder surface, one using the hydrogel and the other attached via the silicone adhesive. The $L=6 \mathrm{~mm}$ sensor was chosen to benefit from optimum sensor sensitivity between 0 and $50 \%$ strain. A slight pre-stretch of each sensor was performed during placement to ensure good attachment of the device to the adhesive film. Sensor resistance was measured using an ohmmeter via short, thin $(d=0.12 \mathrm{~mm}) \mathrm{Al}$ wires attached to the sensor contact pads to ensure a robust measurement process. The resistance of each sensor was recorded as a function of increasing bladder volume. Each sensor was attached to the bladder using the hydrogel adhesive, and two of the three sensors were also taped down securely using thin strips of the hydrogel placed perpendicular to the length of the sensor at either end of the sensor's active area. Two sensors were placed in a vertical direction from the neck of the bladder toward the base, and one was placed diagonally between the neck and the base to investigate the effect of sensor placement on the bladder.

\section{ASSOCIATED CONTENT}

\section{S Supporting Information}

The Supporting Information is available free of charge on the ACS Publications website at DOI: 10.1021/acsomega.8b02609.

Six figures/tables showing sensor stretch and cycling steps, $\mathrm{Au}$ crack formation during strain of the sensor, additional photographs depicting biocompatible adhesives stretching capability, additional data taken from in vitro testing on pig's bladder, $\mathrm{Cr} / \mathrm{Au}$ sensor sensitivity data, and tabular review of various adhesives tested on balloon model (PDF)

\section{AUTHOR INFORMATION}

\section{Corresponding Author}

*E-mail: ramuz@emse.fr (M.R.).

\section{ORCID}

Marc Ramuz: 0000-0003-1261-1821

\section{Present Address}

${ }^{\S}$ Dept. of Biomedical Engineering, University of Strathclyde, 40 George Street, Glasgow, G1 1QE, United Kingdom.

\section{Author Contributions}

The article was written through contributions of all authors. All authors have given approval to the final version of the article. S.H. wrote the article. S.H. and M.R. produced the figures. S.H, P.B., and A.R. performed the experiments described. M.R. conceived and supervised the project.

\section{Notes}

The authors declare no competing financial interest.

\section{ACKNOWLEDGMENTS}

The authors would like to acknowledge Dr. Thelma Lovick and Dr. Jonathan Crook of the University of Bristol for fruitful discussions and project guidance. The authors also wish to thank Bastien Marchiori of the Ecole Nationale Supérieure des Mines de Saint-Etienne for assistance with the measurement setup.

\section{REFERENCES}

(1) Irwin, D. E.; Koop, Z. S.; Agatep, B.; Milsom, I.; Abrams, P. Worldwide Prevalence Estimates of Lower Urinary Tract Symptoms, Overactive Bladder, Urinary Incontinence and Bladder Outlet Obstruction. BJU. Int. 2011, 108, 1132-1138.

(2) Milsom, I.; Abrams, P.; Cardozo, L.; Roberts, R. G.; Thüroff, J.; Wein, A. J. How Widespread are the Symptoms of an Overactive Bladder and How Are They Managed? A Population-Based Prevalence Study. BJU. Int. 2001, 87, 760-766.

(3) Willis-Gray, M.; Dieter, A.; Geller, E. Evaluation and Management of Overactive Bladder: Strategies for Optimizing Care. Res. Rep. Urol. 2016, 8, 113-122.

(4) Brown, J. S.; McGhan, W. F.; Chokroverty, S. Comorbidities Associated with Overactive Bladder. Am. J. Manag. Care. 2000, 6, 574-579.

(5) de Groat, W. C.; Yoshimura, N. Afferent Nerve Regulation of Bladder Function in Health and Disease. Handb. Exp. Pharmacol. 2009, 194, 91-138.

(6) Hu, H. Z.; Granger, N.; Jeffery, N. D. Pathophysiology, Clinical Importance, and Management of Neurogenic Lower Urinary Tract Dysfunction Caused by Suprasacral Spinal Cord Injury. J. Vet. Intern. Med. 2016, 30, 1575-1588.

(7) Martens, F. M. J.; Van Kuppevelt, H. J. M.; Beekman, J. A. C.; Rijkhoff, N. J. M.; Heesakkers, J. P. F. A. Limited Value of Bladder Sensation as a Trigger For Conditional Neurostimulation in Spinal Cord Injury Patients. Neurourol. Urodyn. 2010, 29, 395-400.

(8) Chutka, D. S.; Fleming, K. C.; Evans, M. P.; Evans, J. M.; Andrews, K. L. Urinary Incontinence in the Elderly Population. Mayo. Clin. Proc. 1996, 71, 93-101.

(9) Parsons, B. A.; Drake, M. J.; Gammie, A.; Fry, C. H.; Vahabi, B. The Validation of a Functional, Isolated Pig Bladder Model for Physiological Experimentation. Front. Pharmacol. 2012, 3, 1-8.

(10) Wyndaele, J. J.; Gammie, A.; Bruschini, H.; De Wachter, S.; Fry, C. H.; Jabr, R. I.; Kirschner-Hermanns, R.; Madersbacher, H. Bladder Compliance What Does it Represent: Can we Measure it, and is it Clinically Relevant? Neurorol. Urodyn. 2011, 30, 714-722.

(11) Majerus, S. A.; Fletter, P. C.; Ferry, E. K.; Zhu, H.; Gustafson, K. J.; Damaser, M. S. Suburothelial Bladder Contraction Detection with Implanted Pressure Sensor. PloS ONE 2017, 12, 1-16.

(12) Clausen, I.; Tvedt, L. G. W.; Hellandsvik, A.; Rognlien, D. K. W.; Glott, T. An In Vivo MEMS Sensor System for Percutaneous Measurement of Urinary Bladder. 39th. Int. Conf. IEEE Eng. Med. Bio. Soc. (EMBC); Jeju Island: Korea, 2017.

(13) Wang, C.-C.; Huang, C.-C.; Liou, J.-S.; Ciou, Y.-J.; Huang, I.Y.; Li, C.-P.; Lee, Y.-C.; Wu, W.-J. A Mini-Invasive Long-Term Bladder Urine Pressure Measurement ASIC and System. IEEE. Trans. Biomed. Circuits. Syst. 2008, 2, 44.

(14) Cao, H.; Tata, U.; Landge, V.; Li, A.; Peng, Y. B.; Chiao, J. C. A Wireless Bladder Volume Monitoring System Using a Flexible Capacitance-Based Sensor. IEEE Top. Conf. BioWireleSS., Austin, Texas, USA, 2013.

(15) Cong, P.; Suster, M. A.; Chaimanonart, N.; Young, D. J. Wireless Power Recharging for Implantable Bladder Pressure Sensor. IEEE Sens. Conf., Christchurch, New Zealand, 2010

(16) Majerus, S. J. A.; Garverick, S. L.; Suster, M. A.; Fletter, P. C.; Damaser, M. S. Wireless, Ultra-Low-Power Implantable Sensor for Chronic Bladder Pressure Monitoring. J. Emerg. Technol. Comput. Syst. 2012, 8, 1-13.

(17) Ghaffarzadeh, K.; Hayward, J.; He, X. Stretchable and Conformal Electronics 2018-2028, https://www.idtechex.com/ research/reports/stretchable-and-conformal-electronics-2018-2028000593.asp. (accessed September, 2018).

(18) Cai, L.; Song, L.; Luan, P.; Zhang, Q.; Zhang, N.; Gao, Q.; Zhao, D.; Zhang, X.; Tu, M.; Yang, F.; Zhou, W.; Fan, Q.; Luo, J.; Zhou, W.; Ajayan, P. M.; Xie, S. Super-Stretchable, Transparent Carbon Nanotube-Based Capacitive Strain Sensors for Human Motion Detection. Sci. Rep. 2013, 3, 1-8.

(19) Kettlgruber, G.; Kaltenbrunner, M.; Siket, C. M.; Moser, R.; Graz, I. M.; Schwödiauer, R.; Bauer, S. Intrinsically Stretchable and 
Rechargeable Batteries for Self-Powered Stretchable Electronics. J. Mater. Chem. A 2013, 1, 5505-5508.

(20) Kim, R.-H.; Kim, D.-H.; Xiao, J.; Kim, B. H.; Park, S.-I.; Panilaitis, B.; Ghaffari, R.; Yao, J.; Li, M.; Liu, Z.; Malyarchuk, V.; Kim, D. G.; Le, A.-P.; Nuzzo, R. G.; Kaplan, D. L.; Omenetto, F. G.; Huang, Y.; Kang, Z.; Rogers, J. A. Waterproof AlInGaP optoelectronics on stretchable substrates with applications in biomedicine and robotics. Nat. Mater. 2010, 9, 929-937.

(21) Ruh, D.; Reith, P.; Sherman, S.; Theodor, M.; Ruhhammer, J.; Seifert, A.; Zappe, H. Stretchable Optoelectronic Circuits Embedded in a Polymer Network. Adv. Mater. 2013, 26, 1706-1710.

(22) Sekitani, T.; Noguchi, Y.; Hata, K.; Fukushima, T.; Aida, T.; Someya, T. A Rubberlike Stretchable Active Matrix Using Elastic Conductors. Science 2008, 321, 1468-1472.

(23) Khan, Y.; Ostfeld, A. E.; Lochner, C. M.; Pierre, A.; Arias, A. C. Monitoring of Vital Signs with Flexible and Wearable Medical Devices. Adv. Mater. 2016, 28, 4373-4395.

(24) Gao, W.; Emaminejad, S.; Nyein, H. Y. Y.; Challa, S.; Chen, K.; Peck, A.; Fahad, H. M.; Ota, H.; Shiraki, H.; Kiriya, D.; Lien, D.-H.; Brooks, G. A.; Davis, R. W.; Javey, A. Fully Integrated Wearable Sensor Arrays for Multiplexed In Situ Perspiration Analysis. Nature 2016, 529, 509-514.

(25) Han, Y.; Dong, J. Electrohydrodynamic (EHD) Printing of Molten Metal Ink for Flexible and Stretchable Conductor with SelfHealing Capability. Adv. Mater. Tech. 2017, 3, 1700268.

(26) Kang, S.-J.; Pak, J.-J. A Review: Flexible, Stretchable Multifunctional Sensors and Actuators for Heart Arrhythmia Therapy. Micro \& Nano Syst. Lett. 2017, 5, 1-8.

(27) Viventi, J.; Kim, D.-H.; Vigeland, L.; Frechette, E. S.; Blanco, J. A.; Kim, Y.-S.; Avrin, A. E.; Tiruvadi, V. R.; Hwang, S.-W.; Vanleer, A. C.; Wulsin, D. F.; Davis, K.; Gelber, C. E.; Palmer, L.; Van der Spiegel, J.; Wu, J.; Xiao, J.; Huang, Y.; Contreras, D.; Rogers, J. A.; Litt, B. Flexible, Foldable, Actively Multiplexed, High-Density Electrode Array for Mapping Brain Activity In Vivo. Nature NeuroSci 2011, 14, 1599-1605.

(28) Zhang, S.; Hubis, E.; Girard, C.; Kumar, P.; DeFranco, J.; Cicoira, F. Water Stability and Orthogonal Patterning of Flexible Micro-Electrochemical Transistors on Plastic. J. Mater. Chem. C 2016, 4, 1382-1385.

(29) Zhang, S.; Hubis, E.; Tomasello, G.; Soliveri, G.; Kumar, P.; Cicoira, F. Patterning of Stretchable Organic Electrochemical Transistors. Chem. Mater. 2017, 29, 3126-3132.

(30) Wang, X.; Zhang, H.; Yu, R.; Dong, L.; Peng, D.; Zhang, A.; Zhang, Y.; Liu, H.; Pan, C.; Wang, Z. L. Dynamic Pressure Mapping of Personalized Handwriting by a Flexible Sensor Matrix Based on the Mechanoluminescence Process. Adv. Mater. 2015, 27, 2324-2331.

(31) Shimojo, M.; Namiki, A.; Ishikawa, M.; Makino, R.; Mabuchi, K. A Tactile Sensor Sheet Using Pressure Conductive Rubber with Electrical-Wires Stitched Method. IEEE Sens. J. 2004, 4, 589-596.

(32) Zhou, J.; Yu, H.; Xu, X.; Han, F.; Lubineau, G. Ultrasensitive, Stretchable Strain Sensors Based on Fragmented Carbon Nanotube Papers. ACS Appl. Mater. Interfaces 2017, 9, 4835-4842.

(33) Cai, G.; Wang, J.; Qian, K.; Chen, J.; Li, S.; Lee, P. S. Extremely Stretchable Strain Sensors Based on Conductive Self-Healing Dynamic Cross-Links Hydrogels for Human-Motion Detection. Adv. Sci. 2016, 4, 1600190.

(34) Guo, Y.; Li, Y.-H.; Guo, Z.; Kim, K.; Chang, F.-K.; Wang, S. Bio-Inspired Stretchable Absolute Pressure Sensor Network. Sensors 2016, 16, 55.

(35) Yoshimoto, S.; Araki, T.; Uemura, T.; Noda, Y.; Sekitani, T. Flexible Electronics for Bio-Signal Monitoring in Implantable Applications. IEICE Elecs. Exp. 2017, 14, 20172003.

(36) Khodagholy, D.; Gelinas, J. N.; Thesen, T.; Doyle, W.; Devinsky, O.; Malliaras, G. G.; Buzsáki, G. NeuroGrid: Recording Action Potentials from the Surface of the Brain. Nat. NeuroSci. 2014, $18,310-315$.

(37) Kim, D.-H.; Viventi, J.; Amsden, J. J.; Xiao, J.; Vigeland, L.; Kim, Y.-S.; Blanco, J. A.; Panilaitis, B.; Frechette, E. S.; Contreras, D.; Kaplan, D. L.; Omenetto, F. G.; Huang, Y.; Hwang, K.-C.; Zakin, M.
R.; Litt, B.; Rogers, J. A. Dissolvable Films of Silk Fibroin for Ultrathin Conformal Bio-Integrated Electronics. Nat. Mater. 2010, 9, 511-517.

(38) Viventi, J.; Kim, D.-H.; Moss, J. D.; Kim, Y.-S.; Blanco, J. A.; Annetta, N.; Hicks, A.; Xiao, J.; Huang, Y.; Callans, D. J.; Rogers, J. A.; Litt, B. A Conformal, Bio-Interfaced Class of Silicon Electronics for Mapping Cardiac Electrophysiology. Sci. Transl. Med. 2010, 2, 1-9.

(39) Rajagopalan, S.; Sawan, M.; Ghafar-Zadeh, E.; Savadogo, O.; Chodavarapu, V. A Polypyrrole-Based Strain Sensor Dedicated to Measure Bladder Volume in Patients with Urinary Dysfunction. Sensors 2008, 8, 5081-5095.

(40) Lacour, S. P.; Wagner, S.; Huang, Z.; Suo, Z. Stretchable Gold Conductors on Elastomeric Substrates. Appl. Phys. Lett. 2003, 82, 2404-2406.

(41) Hole, J. W. Human Anatomy and Physiology, 1st ed.; Wm. C. Brown, 1984.

(42) Wang, C.-C.; Huang, C.-C.; Liou, J.-S.; Ciou, Y.-J.; Huang, I.Y.; Li, C.-P.; Lee, Y.-C.; Wu, W.-J. A Mini-Invasive Long-Term Bladder Urine Pressure Measurement ASIC and System. IEEE Trans. Biomed. And Systs. 2008, 2, 44-49.

(43) Nenadic, I.; Mynderse, L.; Husmann, D.; Mehrmohammadi, M.; Bayat, M.; Singh, A.; Denis, M.; Urban, M.; Alizad, A.; Fatemi, M. Noninvasive Evaluation of Bladder Wall Mechanical Properties as a Function of Filling Volume: Potential Application in Bladder Compliance Assessment. PLOS One 2016, 11, e0157818.

(44) Polymer Science INC. P. S.-2066 Technical Datasheet, http:// www.polymerscience.com/wp-content/uploads/2016/12/PS-2066092016.pdf. (accessed November, 2018).

(45) Polymer Science INC. PS-1446 Technical Datasheet, http:// www.polymerscience.com/datasheets/P-DERM/PS-1446_Hydrogel. pdf. (accessed November, 2018). 